\title{
Are Relativistic Effects Significant For The Analysis Of Whistler-Mode Waves In The Earth's Magnetosphere?
}

\author{
S.S. SAZHIN \\ Fluent Europe Ltd, Hutton's Buildings, 146 West Street, Sheffield S1 $4 E S$, U.K. \\ A.E. SUMNER \\ Physics Department, University of Canterbury, Christchurch, New Zealand \\ N.M. TEMME \\ Centrum voor Wiskunde en Informatica, Kruislaan 419 \\ $1098 \mathrm{SJ}$, Amsterdam, The Netherlands
}

\begin{abstract}
It is pointed out that relativistic effects lead to an increase in whistler-mode refractive index and to a decrease in the range of frequencies for which instability of these waves occurs. The latter effect is particularly important in a rarefied plasma in a strong magnetic field where the electron plasma frequency $(\Pi)$ is of the order of or smaller than the electron gyrofrequency $(\Omega)$. The condition $\Pi \lesssim \Omega$ is satisfied in some regions of the Earth's magnetosphere (e.g. outside the plasmasphere near the magnetospheric equator) and in these regions the analysis of whistler-mode waves requires the weakly relativistic rather than the nonrelativistic approach even if the thermal velocities of magnetospheric particles are well below the velocity of light.
\end{abstract}

\section{INTRODUCTION}

Since the pioneering paper by Storey (1953) there have been different developments in the theory of whistler-mode waves. In particular, attempts have been made to take into account both non-zero electron temperature and anisotropy of the electron distribution function and their effects on whistler-mode propagation, growth or damping [ e.g. Sazhin, 1993a]. In most cases the theory of whistler-mode propagation in a hot anisotropic plasma has been based on the nonrelativistic approximation, i.e. on a self-consistent solution of the linearized Maxwell equations and the nonrelativistic Vlasov equation. This approximation was believed to be applicàlic with conficience to magnetospheric conditions, which satisfy

$$
w \ll c
$$

where $w$ is the thermal velocity of electrons, and $c$ is the velocity of light. At the same time, most of those who

Space Plasmas: Coupling Between Small and Medium Scale Processes

Geophysical Monograph 86

Copyright 1995 by the American Geophysical Union developed this theory seem to have ignored the fact that the nonrelativistic approach to the analysis of waves in a hot plasma is not self-consistent, as it neglects relativistic effects in the Vlasov equation, but takes them into account in the Maxwell equations, which are relativistic by their nature.

An alternative approach to the theory of whistler-mode waves in a hot plasma has been based on the so called weakly relativistic approximation which uses condition (1) with terms of the order of $w^{2} / c^{2}$ taken into account, allowing substantial simplification of the general relativistic wave dispersion equation. However, even this relatively simple weakly relativistic whistler-mode dispersion equation is much more complicated than the nonrelativistic dispersion equation, and its applications have been very limited [e.g. Robinson, 1987].

In a series of papers reviewed by Sazhin (1993a) [see also the most recent publications: Sazhin et al., 1993; Sazhin, 1993b] we attempted to develop an asymptotic theory of whistler-mode propagation, instability or damping in a weakly relativistic plasma. Restricting ourselves to a limiting, but practically important case of whistler-mode propagation strictly parallel to the magnetic field we obtained some approximate solutions to the weakly relativistic dispersion equation. These solutions are rather simple and we hope that they will be widely used in the plasma physics 
community. The main restriction of these solutions is that we cannot always be sure of the range of their applicability, just as we cannot always be sure of the range of applicability of solutions to the nonrelativistic dispersion equation.

In order to specify the range of applicability of different approximate solutions to the weakly relativistic dispersion equation, as well as those of the nonrelativistic equation, we need to compare them with the results of a rigorous numerical analysis of the weakly relativistic whistler-mode dispersion equation. Such a comparison for parallel whistlermode waves was' made by Sazhin et al (1993a). In what follows we briefly discuss the main ideas of this paper with particular emphasis to magnetospheric applications of the obtained results.

\section{BASIC EQUATIONS}

The dispersion equation for parallel whistler-mode waves in a weakly relativistic (condition (1) is valid) anisotropic plasma can be written as [Sazhin et al., 1992]:

$$
N^{2}=1-\frac{2 X}{r}\left[\mathcal{F}_{1 / 2,2}-\frac{d \mathcal{F}_{3 / 2,2}}{d z}\left(A_{e}-1\right) N^{2}\right],
$$

where:

$\mathcal{F}_{q, p} \equiv \mathcal{F}_{q, p}(z, a, b)=-i \int_{0}^{\infty} e^{i z t-\frac{a z^{2}}{1-i t}}(1-i t)^{-q}(1-i b t)^{-p} d t$

$z=2(1-Y) / r ; a=N^{2} / r ; r=p_{0 \|}^{2} /\left(m_{e}^{2} c^{2}\right) ; b=A_{e} ;$ $X=\Pi_{0}^{2} / \omega^{2} ; Y=\Omega_{0} / \omega ; A_{e}=p_{0 \perp}^{2} / p_{0 \|}^{2} ; \Pi_{0}, \Omega_{0}$ and $\omega$ are the electron plasma frequency at rest, the electron gyrofrequency at rest and the wave frequency (which is complex in general) respectively, $N \equiv c k / \omega$ is the wave refractive index, $m_{e}$ is the electron mass at rest, $c$ is the velocity of light, $k$ is the wave number.

When deriving (2) we assumed an electron distribution function of the form:

$$
f\left(p_{\perp}, p_{\|}\right)=\left(\pi^{3 / 2} j ! p_{0 \perp}^{2 j+2} p_{0 \|}\right)^{-1} p_{\perp}^{2 j} \exp \left(-\frac{p_{\perp}^{2}}{p_{0 \perp}^{2}}-\frac{p_{\|}^{2}}{p_{0 \|}^{2}}\right),
$$

where $p_{0 \perp(11)}$ is the electron thermal momentum in the direction perpendicular (parallel) to the magnetic field, $p_{\perp}$ and $p_{\|}$are the electron momenta in the corresponding directions.

In a nonrelativistic limit, $c \rightarrow \infty$, equation (2) can be simplified to:

$$
N^{2}=1+\left(A_{e}-1\right) X+\frac{X}{N \sqrt{r}}\left[A_{e}+\left(1-A_{e}\right) Y\right] Z(\xi)
$$

where

$$
Z(\xi)=i \sqrt{\pi} \exp \left(-\xi^{2}\right)-2 \int_{0}^{\xi} \exp \left(-\xi^{2}+t^{2}\right) d t
$$

$\xi=z / 2 \sqrt{a}$

Solutions to equations (2) and (5) are greatly simplified if we restrict our analysis to the case of weakly growing or weakly damped waves, i.e.

$$
|\gamma| \equiv|\Im \omega|<\min \left(\Re \omega, \Omega_{0}-\Re \omega\right) .
$$

In view of (7) we can present the complex equation (2) as a system of two equations:

$$
\begin{gathered}
N^{2}=1-\frac{2 X}{r}\left[\Re \mathcal{F}_{1 / 2,2}-\frac{d \Re \mathcal{F}_{3 / 2,2}}{d z}\left(A_{e}-1\right) N^{2}\right] \\
\tilde{\gamma}=\frac{X\left[\Im \mathcal{F}_{1 / 2,2}\left(1+\left(A_{e}-1\right) N^{2}\right)-\left(A_{e}-1\right) N^{2} \Im \mathcal{F}_{3 / 2,2}\right]}{r\left[1+\frac{2 X}{r^{2}} \sum_{i=0}^{3} \tilde{a}_{i} \Re \mathcal{F}_{i-\frac{1}{2}, 2}\right]}
\end{gathered}
$$

where

$$
\tilde{a}_{0}=Y+N^{2}\left(1+Y\left(A_{e}-1\right)\right)+N^{4}\left(A_{e}-1\right),
$$

$$
\begin{gathered}
\tilde{a}_{1}=\left(A_{e}-1\right) N^{2} r-Y-2 N^{2}\left(1+Y\left(A_{e}-1\right)\right)-3 N^{4}\left(A_{e}-1\right), \\
\tilde{a}_{2}=-\left(A_{e}-1\right) N^{2} r+N^{2}\left(1+Y\left(A_{e}-1\right)\right)+3 N^{4}\left(A_{e}-1\right), \\
\tilde{a}_{3}=-\left(A_{e}-1\right) N^{4}, \\
\tilde{\gamma} \equiv \frac{\gamma}{\omega} .
\end{gathered}
$$

To simplify the notation we hereafter assume that $\omega \equiv$ $\Re \omega$.

In a similar way we can reduce the complex nonrelativistic dispersion equation (5) to the following system of equations:

$$
\begin{gathered}
N^{2}=1+\left(A_{e}-1\right) X+\frac{X}{N \sqrt{r}}\left[A_{e}+\left(1-A_{e}\right) Y\right] \Re Z\left(\xi_{0}\right), \\
\tilde{\gamma} \equiv \frac{\gamma}{\omega}=\frac{-\sqrt{\pi}\left[A_{e}-\left(A_{e}-1\right) Y\right] \exp \left(-\xi_{0}^{2}\right)}{\frac{2(1-Y)}{X \xi_{0}}+\kappa \xi_{0}+\Re Z\left(\xi_{0}\right)\left[A_{e}+\kappa \xi_{0}^{2}\right]},
\end{gathered}
$$

where

$$
\xi_{0} \equiv \Re \xi, \quad \kappa=\frac{2\left[A_{e}+Y\left(1-A_{e}\right)\right]}{Y-1} .
$$

Equations (8) and (10) describe wave propagation, while equations (9) and (11) describe wave growth $(\tilde{\gamma}>0)$ or damping $(\tilde{\gamma}<0)$.

In the cold plasma limit $\left(\left|\xi_{0}\right| \rightarrow \infty\right)$ equation (10) reduces to:

$$
N=N_{00} \equiv \sqrt{1+\frac{X}{Y-1}},
$$

while equation (11) gives in the same limit: $\tilde{\gamma}=0$. This means that in a cold plasma whistler-mode waves propagate without damping or growth. In the limiting case $Y \gg 1$ and $X \gg 1$ equation (12) reduces to that derived and analized by Storey (1953).

In the limit $r \rightarrow 0$, but keeping terms of the order of $r$, we can write an approximate solution of (8) as (Sazhin, 1993a):

$$
N=N_{00}\left[1+\frac{\beta_{e} Y^{3}}{2(Y-1)^{3}}+\frac{\beta_{e}\left(1+4 A_{e}\right) Y^{2}}{4(Y-1)^{2} N_{00}^{2}}-\frac{\beta_{e} A_{e} Y^{2}}{2(Y-1)^{2}}\right] \text {, }
$$




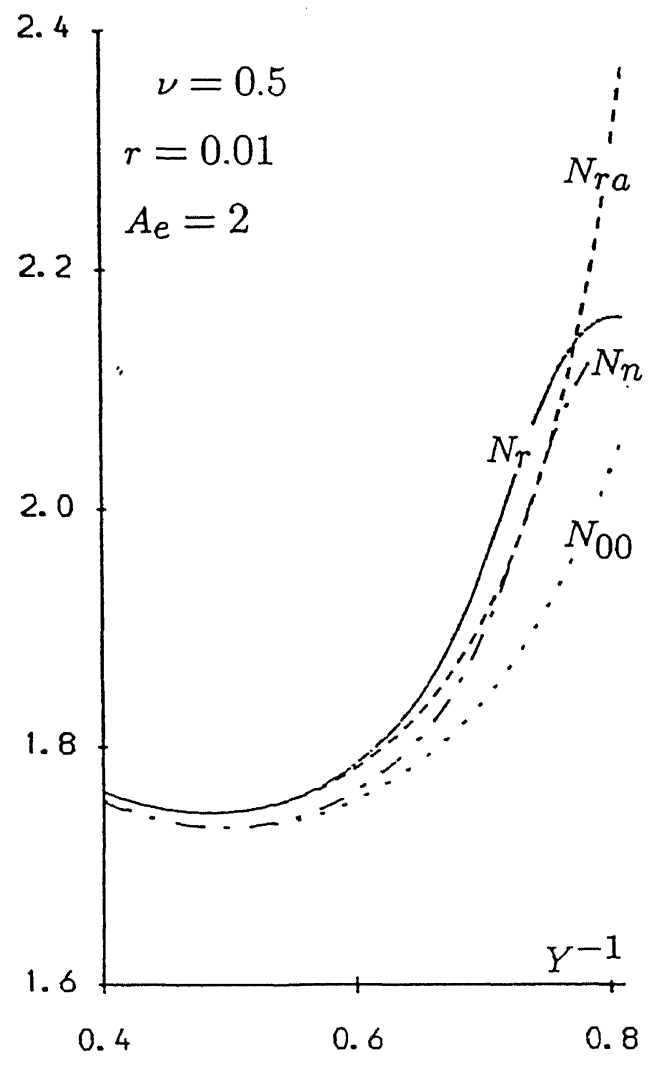

Fig 1. Plots of $N_{r}$ versus $Y^{-1} \equiv \omega / \Omega_{0}$, solid, (see equation (8)), $N_{n}$ versus $Y^{-1} \equiv \omega / \Omega_{0},-\cdots-\cdots-($ see equation $(10)), N_{r a}$ versus $Y^{-1} \equiv \omega / \Omega_{0}, \ldots-\cdots$, (see equation $\left.(13)\right), N_{00}$ versus $Y^{-1} \equiv \omega / \Omega_{0}, \quad-\quad-\quad$ (see equation (12)) for a plasma with the following parameters: $r=0.01, \nu=0.5$ and $A_{c}=2$. Plots are shown only for those $Y^{-1} \equiv \omega / \Omega_{0}$ when $\bar{\gamma}_{r, n, n s} \lesssim 0.2$ (condition (7) is satisfied).

where $\beta_{e}=0.5 \nu r, \nu=\Pi_{0}^{2} / \Omega_{0}^{2}, N_{00}$ is defined by (12). In the limit $N_{00}^{2} \rightarrow \infty$ this expression reduces to that which could be derived from the corresponding nonrelativistic dispersion equation (10). At $A_{c}=1$ it reduces to the expression derived by it Imre (1962)

The nonrelativistic expression for $\tilde{\gamma}$ can be considerably simplified if we neglect the effects of nonzero electron temperature on wave propagation, it is then reduced to:

$$
\tilde{\gamma}=\frac{\sqrt{\pi} \xi_{00}(Y-1)\left[A_{e}-\left(A_{e}-1\right) Y\right] \exp \left(-\xi_{00}^{2}\right)}{\frac{2(1-Y)^{2}}{X}+Y},
$$

where

$$
\xi_{00}=\frac{1-Y}{N_{00} \sqrt{r}}
$$

The values of $N$ which follow from equations (8), (10), (12), (13) and the values of $\tilde{\gamma}$ which follow from equations (9), (11) and (13) will be compared in the next section for different values of the plasma parameters $\left(\nu, r\right.$ and $\left.A_{c}\right)$.

\section{RESULTS}

In this section we compare numerical values of $N$ and $\tilde{\gamma}$, obtained from the approximations discussed in section 2 for different values of the parameters typical for conditions in the Earth's magnetosphere. Firstly we consider a relatively hot $(r=0.01$, which corresponds to $T \approx 2.5 \mathrm{keV})$ and rarefied $(\nu=0.5)$ plasma with anisotropy $A_{c}=2$ (realistic conditions outside the plasmasphere). Plots of $N$ versus $Y^{-1} \equiv \omega / \Omega_{0}$ and $\tilde{\gamma}$ versus $Y^{-1} \equiv \omega / \Omega_{0}$ for the different approximations for this plasma are shown in Figs 1 and 2. As can be seen from Fig 1 , the values of $N_{r a}$, determined by (13), are closest to those of $N_{r}$, determined by (2) at $\omega / \Omega_{0} \lesssim 0.78$. In any case approximation $N_{\text {ra }}$ is better than the nonrelativistic approximation $N_{n}$, determined by (5) in this frequency range. The latter approximation is applicable for the qualitative analysis of $N$ only. The curves $N$ versus $Y^{-1} \equiv \omega / \Omega_{0}$ are hereafter presented only for those $\omega / \Omega_{0}$ at which $|\tilde{\gamma}| \lesssim 0.2$.

The curves $\bar{\gamma}_{n s}$, determined by (14), and $\tilde{\gamma}_{n}$, determined by (11), shown in Fig 2, coincide within the accuracy of plotting, but the deviation between relativisrtic and nonrelativistic curves is quite noticeable. Relativistic theory predicts stronger instability but at a lower frequency. The frequency of marginal stability is approximately equal to

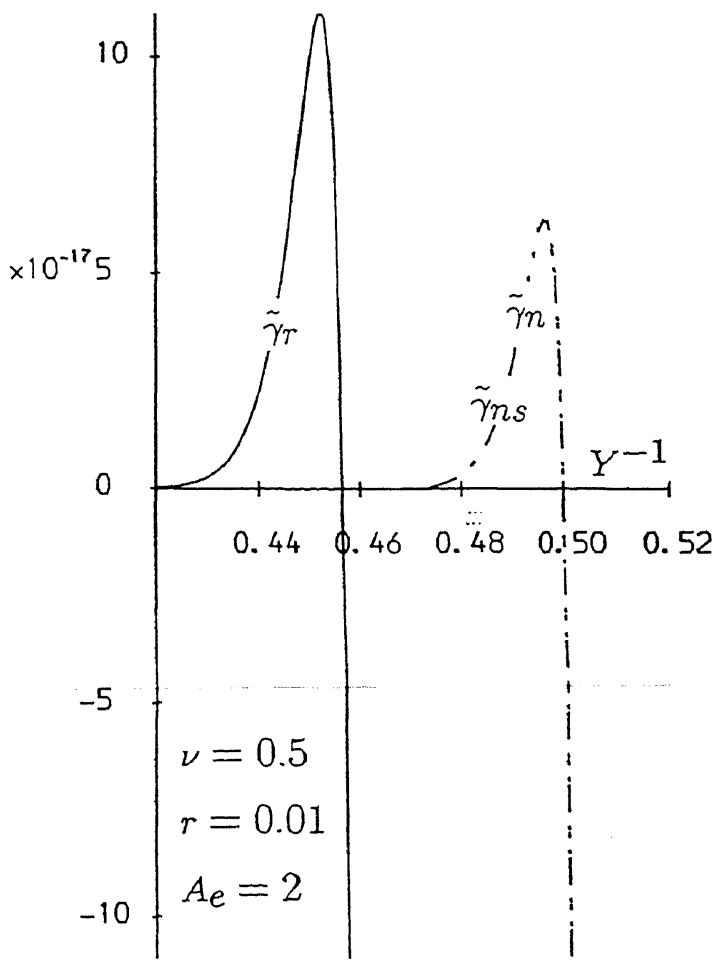

Fig 2. Plots of $\tilde{\gamma}_{r}$ versus $Y^{-1} \equiv \omega / \Omega_{0}$, solid, (see equation (9)), $\bar{\gamma}_{n}$ versus $Y^{-1} \equiv \omega / \Omega_{0},-\cdots-\cdots-,($ see equation $(11)), \bar{\gamma}_{n s}$ versus $Y^{-1} \equiv \omega / \Omega_{0},-\ldots$ same plasma parameters as in Fig 1 . 
$0.457 \Omega_{e}$, which is consistent with the results of asymptotical analysis (Sazhin et al., 1992).

Curves similar to those shown in Figs 1 and 2 were presented by Sazhin et al (1992) for other values of parameters $\left(\nu=1, r=0.01, A_{c}=2 ; \nu=5, r=0.01, A_{c}=2\right.$; $\nu=1, r=0.004, A_{e}=2 ; \nu=1, r=0.004, A_{e}=1$; $\nu=1, r=0.004, A_{e}=3$ ). We tried to choose values of the parameters $r, \nu$ and $A_{c}$, used in the computations, relevant to the conditions in the Earth's magnetosphere. However, these parameters vary there over such a wide range that we were bound to restrict ourselves to some illustrative examples only. The results of our analysis of these curves allowed us to draw the following conclusions.

1) Expression (13) for $N$ is the best approximation for numerical values of $N$ obtained in a weakly relativistic limit at relatively low frequencies (roughly $Y^{-1} \equiv \omega / \Omega_{0} \lesssim 0.6$ ), but it breaks down rapidly as the wave frequency approaches the electron gyrofrequency (roughtly $Y^{-1} \equiv \omega / \Omega_{0} \gtrsim 0.6$ ). In a dense plasma, where $N_{00} \gg 1$, the contribution of the relativistic term in (13) (the term proportional to $N_{00}^{-2}$ in square brackets) is negligible, while in a rarefied plasma, where $N_{00}$ is close to unity, the contribution of this term can be of the same order of magnitude or larger than the contribution of other terms.

2) The nonrelativistic expression for $N$ following from equation (10) can be used for qualitative analysis of whistlermode propagation in a weakly relativistic plasma; this expression is the best approximation to the corresponding weakly relativistic expression when the wave frequency is close to the electron gyrofrequency, provided condition (7) is satisfied.

3) Relativistic effects lead to a decrease in the frequency range of instability, and the magnitude of this effect increases as $\nu$ decreases.

4) For moderate values of anisotropy $\left(T_{\perp} / T_{\|} \approx 2\right)$ relativistic effects lead to an increase in the maximum value of the increment of instability $\left(\gamma_{\max }\right) ; \gamma_{\max }$ increases rapidly with increasing electron temperature and aniso

5) Expression (14) for the increment of $w$ instability (or the decrement of damping) is a $g$ imation to the nonrelativistic expression (11) i.e. when we have instability; expression (14) pre damping of whistler-mode waves at frequencies electron gyrofrequency, when compared with th of expression (11).

Acknowledgements. The authors are grateful $t$ for their helpful comments (special thanks are due referees who drew our attention to the paper by Im of the authors (S.S.) would like to thank NERC support at the initial stage of this project.

\section{REFERENCES}

Imre, K., Oscillations in a relativistic plasma, $P h$. 459-466, 1962.

Robinson, P. ., Thermal effects on parallel-propaga mode waves, J. Plasma Phys., 37, 149-162, 1987

Sazhin, S. S., Whistler-mode waves in a hot plasm. University Press, 1993a.

Sazhin, S. S., A relativistic theory of plasma cutSpace Sci., 203, 317-327, 1993.

Sazhin, S. S., A. E. Sumner, and N. M. Temme and non-relativistic analysis of whistler-mode $\mathbf{w}$ anisotropic plasma, J. Plasma Phys, 47, 163-174

Sazhin, S. S., A. E. Sumner, N. M. Temme ar An approximate solution of the parallel whistle dispersion equation in a weakly relativistic pla Phys. Control. Fusion, 95, 117-126, 1993.

Storey, L. R. O., An investigation of whistling atmos Trans. Roy. Soc (London), A246, 113-141, 1953.

S. S. Sazhin, Fluent Europe Ltd., Hutton's Buildir Street, Sheffield, S1 4ES, U.K.

A. E. Sumner, Physics Department, University of Christchurch, New Zealand.

N. M. Temme, Centrum voor Wiskunde en Inform laan 413, 1098 SJ, Amsterdam, The Netherlands. 